\title{
Chloroquine: An Old to be Repurposed Drug For COVID-19 Infection (Risk and Benefit)
}

\author{
Oki Nugraha Putra*, Ana Khusnul Faizah, Nani Wijayanti Dyah Nurrahman, Hardiyono \\ Study Program of Pharmacy, Faculty of Medicine, Hang Tuah University, Surabaya, Indonesia
}

\begin{abstract}
Severe acute respiratory syndrome coronavirus 2 (SARS-CoV-2) causes Coronavirus Disease 2019 (COVID-19) with a high rate of mortality. Chloroquine and hydroxychloroquine have been used for antimalarial and autoimmune diseases for many years. Due to low toxicity and well tolerability as well as immunomodulatory properties, these drugs are proposed to treat viral infection. Some studies, both in vitro and in a clinical setting, have been evaluated for their ability to treat SARS$\mathrm{CoV}-2$ as promising therapies. Although The National Agency of Drugs and Food Control of The Republic of Indonesia issued emergency authorization for chloroquine and hydroxychloroquine to be used against COVID-19 infection, the efficacy of these drugs is still based on small clinical nonrandomized trials with a limited number of patients. However, the use of these drugs without any risks. The safety of these drugs to be used in COVID-19 patients is lacking. Some experts noticed that the drugs cause harmful adverse effects, especially a harmful QT prolongation. In Indonesia, until now, no study evaluates the effectiveness as well as the safety of these drugs to be used in COVID-19 infection. This article will discuss the role of chloroquine or hydroxychloroquine and its safety to be used against COVID-19 infection.
\end{abstract}

Keywords: SARS-CoV-2; COVID-19; Chloroquine; Hydroxychloroquine

*corresponding author

Email: oki.nugraha@hangtuah.ac.id

\section{INTRODUCTION}

In late December 2019, the novel virus called severe acute respiratory syndrome coronavirus-2 (SARSCoV-2) was found in Wuhan, China. A large number of patients were found with pneumonia with unknown etiology and seemed not responsive to standard therapy of pneumonia. It is known that the virus was transmitted from animals to humans or as zoonotic ( $\mathrm{Lu}$ et al., 2020). In March 2020, the WHO stated that COVID-19 virus infection became a global pandemic with many countries all over the world, reporting positive cases of confirmed patients with COVID-19 (WHO, 2020). In Indonesia, the first case of COVID-19 reported in March 2020. On May $21^{\text {st }}, 2020$, there were significant cases of COVID-19 positive patients of more than 20.000 cases, with the mortality rate was $6.3 \%$, the highest of all Southeast Asia countries (Kemenkes RI, 2020). Many programs have carried out by the Indonesian government, including washing hands with soap as often as possible, using hand sanitizer, physical distancing, learning, and working from home, using masks when leaving home, is a way to break the spread of the COVID-19 virus chain. At present, the spread of SARS-CoV-2 from human to human is the main transmission via airborne droplets to the nasal mucosal or through the eyes from symptomatic patients (Han et al., 2020).
SARS-CoV-2 causes symptoms or without symptoms when someone infected with the virus. The most common symptoms are fever, muscle aches, cough, and respiratory symptoms such as dyspnea (Guo et al., 2020). Symptoms worsen in the elderly, smoking, and patients with underlying or chronic diseases such as diabetes, cancer, heart disease, and autoimmune diseases. It has been proposed that smoking habits, diabetes, and cardiovascular disease increase the expression of angiotensin-converting enzyme-2 (ACE-2) receptors (Fang et al., 2020). The problem that arises from COVID-19 infection is to choose a therapy that effective and safe. Until now, the food and drug administration (FDA) has not yet approved the best drugs to manage COVID-19 infections. A large number of drugs given to patients, only limited to case reports in various countries. To the best of our knowledge, there are no randomized controlled clinical trials with many patients and the best methods that evaluate the effectiveness and safety of a particular drug for COVID-19 infection. Antimalaria drugs, namely chloroquine and hydroxychloroquine, have been reported to be used to treat COVID-19 infections in several countries all over the world. Indonesia has even imported Favipiravir $\left(\operatorname{Avigan}^{\circledR}\right)$ from Japan and ordered a pharmaceutical company to produce millions of chloroquine tablets to overcome COVID-19 infections in Indonesia. Chloroquine has been known for a long time as an antimalarial drug. WHO is planning 
a multinational clinical trial, not blinded, related to COVID-19 called SOLIDARITY. The study divided into four groups, namely the lopinavir/ritonavir (LPV/r) group and IFN- $\beta$, the LPV/ $r$ group, the chloroquine, or chloroquine group and the remdesivir group. Although The National Agency of Drugs and Food Control of The Republic of Indonesia has granted emergency authorization for using antimalarial drugs, chloroquine and hydroxychloroquine to treat COVID-19 infection in Indonesia, unfortunately, until now there has been no clinical studies that evaluate the effectiveness and safety of chloroquine to treat COVID-19 infection in Indonesia. This review article will discuss at a glance the role of chloroquine and its safety as therapy for COVID-19 infection.

\section{METHODS}

The method to review this article is a narrative review study. In contrast to systematic reviews, a narrative study is not necessary a systematic, structured, and validated library search method. Therefore, the article selection flow is not absolutely displayed (Pae, 2015). This narrative study used published articles from the library search process using a scientific research database, PubMed. Keywords, such as SARS-CoV-2, COVID-19, chloroquine, hydroxychloroquine, and QT prolongation, are used to search libraries. Articles used in this study are no more than the last ten years. Literature search as a reference in published articles (i.e. SCOPUS) is also used in this study.

\section{RESULTS AND DISCUSSION}

\section{What is Chloroquine?}

Chloroquine and hydroxychloroquine are 4-aminoquinoline synthetic antimalarial drugs, originated from the bark of cinchona. Chloroquine has been used for the treatment and prophylaxis of malaria for many years. Nowadays, chloroquine and hydroxychloroquine cannot be used any longer for the treatment of malaria disease due to the high-level resistance of malaria parasites. These drugs are commonly used for autoimmune disease or as disease-modifying anti-rheumatic drugs (DMARDs), which is widely used to treat systemic lupus erythematosus (SLE) and rheumatoid arthritis (RA), due to strong immunomodulatory capacity (Lee et al., 2011). Because of low toxicity, inexpensive, well tolerably, and immunomodulatory characteristics, chloroquine, and hydroxychloroquine have also been considered to treat viral infections. Since chloroquine and hydroxychloroquine are not patented by the pharmaceutical company, it a great advantage to conduct clinical trials about their efficacy in COVID-19 infection. Chloroquine has been known from several studies to have antiviral effects in middle east respiratory syndrome (MERS), severe acute respiratory syndrome (SARS), Ebola, and the latest COVID-19 virus, but without any data showing clinical efficacy (Savarino et al., 2003 ; Cortegiani et al., 2020).

Both of them are soluble in water, but hydroxychloroquine is more soluble than chloroquine due to its N-hydroxyethyl chain. Chloroquine and hydroxychloroquine have a very long elimination half-life ( $\mathrm{t} 1 / 2$ ), 38 days, and 54 days, respectively (Kalia \& Dutz 2007). Because of a very long elimination half-life, they will accumulate into the tissue after chronic exposure to the drugs (Jorge et al., 2018). Chloroquine and hydroxychloroquine are metabolized in the liver by the cytochrome P450 enzyme, and more than $50 \%$ excreted by the kidneys as unchanged drugs. Due to the very long elimination half-life, these drugs are slowly excreted in the urine. Thus, even the therapy has stopped, it is crucial to monitor the possible side effects of these drugs, such as keratopathy and maculopathy. The prevalence of retinopathy after administering chloroquine was lower than $2 \%$ and the incidence of retinopathy is more common in Asian patients (Marmor et al., 2016). There were two aspects to be considered to use chloroquine and hydroxychloroquine to treat COVID-19 infection : (1) the COVID-19 infection is a pandemic, FDA has issued the emergency authorization of chloroquine and hydroxychloroquine to treat COVID-19 infection; therefore millions of infected patients will be administered chloroquine or hydroxychloroquine by the clinician, 2) the dose of these drugs for COVID-19 infection may higher than that of for autoimmune disease (Touret \& de Lamballerie 2020). The recommended dose of chloroquine base for SLE was 3.5-4.0 mg/kg BW/day (150-300 mg/day) (one tablet of $250 \mathrm{mg}$ chloroquine phosphate equal to $150 \mathrm{mg}$ of chloroquine base).

Several mechanisms of chloroquine and hydroxychloroquine have been observed against SARSCov-2 as follows: (a) chloroquine interferes with the glycosylation process with the ACE-2 receptors, as the site of SARS-CoV-2 binds into the host cells, (b) as a weak base, chloroquine increases the $\mathrm{pH}$ of acidic cellular organelles, inhibits the process of endocytosis in the intermediate stage and virion transport, (c) chloroquine prevents the virion assembly process and viral protein synthesis (Savarino et al., 2003).

\section{In Vitro Activity}

Since the chloroquine and hydroxychloroquine have a similar chemical structure and physicochemical properties, it proposed that the mechanisms of these drugs are also similar. Therefore, chloroquine and hydroxychloroquine are interchangeable against COVID-19 in several in vitro studies. Chloroquine and hydroxychloroquine have good bioavailability, very high volume distribution, and relatively high 
concentration in the kidney, spleen, liver, and lung. A preclinical study reported that the highest concentration of chloroquine was found in lung tissue, about 11.8-450 times higher compared with plasma concentration after the administration of single-dose chloroquine (Adelusi et al., 1982). Pre-clinically, it proposed that chloroquine can be used in COVID-19 associated pneumonia. Some in vitro studies that evaluate the effect of chloroquine and hydroxychloroquine against COVID-19 used African green monkey kidney VeroE6 cells. A study by Yao et al., showed that hydroxychloroquine was more potent $\left(\mathrm{EC}_{50}=0.72 \mu \mathrm{M}\right)$ than chloroquine $\left(\mathrm{EC}_{50}=5.47 \mu \mathrm{M}\right)$ to inhibit COVID-19 in vitro. By the physiological based pharmacokinetic (PBPK) models, administer $400 \mathrm{mg}$ hydroxychloroquine sulfate as a loading dose, given orally, and followed by $200 \mathrm{mg}$ twice daily for four days as a maintenance dose considered to treat COVID-19 infection. The aim of the maintenance dose was to maintain the drug concentration to effective in infected lung tissue of COVID-19. The administration of loading dose and followed by the maintenance dose, it three times the potency of chloroquine when administered with a dose $500 \mathrm{mg}$, twice daily for 5 days (Yao et al., 2020), but, it is unclear the site of action of chloroquine in lung tissue to treat COVID-19 associated pneumonia. Wang et al., stated that the value of EC90 of chloroquine against COVID-19 in Vero E6 cells was $6.90 \mu \mathrm{M}$. This in vitro $\mathrm{EC}_{90}$ of chloroquine is similar to the plasma level when a patient was given $500 \mathrm{mg}$ of chloroquine to treat rheumatoid arthritis (RA) (Wang et al., 2019). A study by Liu et al, reported that the value of cytotoxic concentration (CC50) of chloroquine and hydroxychloroquine was 273.20 and $249.50 \mu \mathrm{M}$, respectively. The selectivity index (CC50/EC50) of chloroquine was higher than that of hydroxychloroquine. It indicated that the activity of hydroxychloroquine to treat COVID-19 was less potent compared to chloroquine (Liu et al., 2020).

\section{Clinical Studies}

In spite of lack of evidence, guidelines from several countries propose chloroquine and hydroxychloroquine to be considered for use in patients with COVID-19. Chinese guidelines recommended chloroquine phosphate $500 \mathrm{mg}$ twice daily for seven days (National Health Commission and State Administration of Traditional Chinese Medicine, 2020). The Italian Society of Infectious Diseases recommends $500 \mathrm{mg}$ of chloroquine phosphate or $200 \mathrm{mg}$ hydroxychloroquine sulfate twice daily for 10 days regardless of severity, but not for prophylactic use (Gruppo collaborativo COVID-19 Lombardia, 2020).

A study by Gautret et al., showed that the administration of hydroxychloroquine with the dose of $200 \mathrm{mg}$, three times daily for ten days along with Azitromycin, is effective for clearing COVID-19 in nasopharyngeal in most patients for three to six days (Gautret et al., 2020). A limitation of the study by Gautret et al., was a small sample size $(n=80)$ without a control group, and the study design was non-randomized and non-blinded. Another limitation was $92 \%$ of patients with mild severity of illness, and there was no comparison group. Therefore, their results can not be interpreted in clinical settings. A study by Yao et al., showed that the infected COVID-19 patients could be administered hydroxychloroquine of $400 \mathrm{mg}$ twice daily for the first day and followed at the dose of $200 \mathrm{mg}$ twice daily for 4 days as a maintenance dose (Yao et al., 2020). Similar to Gautret's work, no placebo was used in this study. Outcomes were not clear, and patients with underlying disease or comorbid were not reported, and their might as cofounding variables that interrupt the main findings. Chloroquine, as a DMARD, has several immunomodulatory properties by suppressing IL- 6 release and tumor necrosis factor- $\alpha$ $(\mathrm{TNF}-\alpha)$. It may prevent the occurrence of cytokine storms that worsen the prognosis of patients with COVID-19 infection (Guo et al., 2020). Another study in a multicenter clinical trial, including more than 100 patients, found that chloroquine has some efficacy to treat COVID-19 infection with pneumonia in China (Gao et al., 2020). This result, without any limitations, the regimentation dose of chloroquine was not clearly stated, poor methodology, and statistical analysis were not included in this study.

A cohort study by Borba et al., analyzes the effect of high dose (600 mg, twice daily for ten days) versus low dose (450 mg twice daily on day one, and once daily for four days) chloroquine as adjunctive therapy for severe COVID-19 infection. The results of this study stated that the administration of a high dose of chloroquine should not be recommended for severe patients with COVID-19 infection due to its serious safety hazards, QT prolongation compared with the low dose of chloroquine. The QT prolongation after the administration of high dose chloroquine will be greater when taken together with oseltamivir and azithromycin. The overall conclusion of this study that it can not be generalized to nonsevere COVID-19 infection (Borba et al., 2020).

Indonesian Society of Respirology has included chloroquine and hydroxychloroquine in therapy for patients who have confirmed positive COVID-19 with mild to severe symptoms. For mild and moderate symptoms, the Indonesian Society of Respirology suggested chloroquine phosphate $500 \mathrm{mg}$, twice daily for 5 days or hydroxychloroquine $400 \mathrm{mg}$, once daily for 5 days. For severe symptoms, the dose of chloroquine phosphate was $500 \mathrm{mg}$, twice daily for day 1 until day 3 , followed by $250 \mathrm{mg}$ of chloroquine phosphate, twice daily for day 4 until day 10 or hydroxychloroquine 400 
mg, once daily for 5 days. For moderate and severe symptoms, azithromycin with dose $500 \mathrm{mg}$ was added once daily for 3 days. Indonesian Society of Respirology also issued a warning that the combination of chloroquine phosphate and azithromycin in some cases caused the QT interval to be prolonged (Indonesian Society of Respirology, 2020).

Acute respiratory distress syndrome (ARDS) is a leading cause of death in COVID-19 patients. The cause of ARDS in SARS-CoV-2 infection is a cytokine storm, which is an uncontrolled systemic inflammatory response due to the release of large amounts of proinflammatory cytokines (IFN- $\alpha$, IFN- $\gamma$, IL-6, TNF- $\alpha$, and TGF $\beta$ ) and large amounts of chemokines (Li et al., 2020). This excessive immune response can cause lung damage and fibrosis, resulting in functional disability (Zumla et al., 2020). Because chloroquine has several immunomodulatory effects, through inhibition of TNF alpha and IL-6, it can prevent cytokine storms that lead to worsening symptoms in COVID-19 patients (Guo et al., 2020).

\section{Safety, Adverse Effects and Risk Factors}

The common adverse effects of chloroquine and hydroxychloroquine in the usual dose are gastrointestinal intolerance, such as nausea, vomiting, and diarrhea (Srinivasa et al., 2017). The loading dose of 800 $\mathrm{mg}$ or higher is associated with serious side effects. One of the side effects that irreversible and severe is retinopathy. Retinopathy is associated with a dose of more than $5 \mathrm{mg} / \mathrm{kg}$ and long treatment of chloroquine and hydroxychloroquine, usually more than 5 years. Cardiomyopathy is the most serious adverse effect of the use of chloroquine and hydroxychloroquine. Hydroxychloroquine was found to be less toxic than chloroquine (Liu et al., 2020). The American Academy of Ophthalmology reported that the major risk factors to retinopathy were hydroxychloroquine with dose more 5 $\mathrm{mg} / \mathrm{kg} /$ actual body weight or chloroquine more $2.3 \mathrm{mg} /$ $\mathrm{kg} /$ actual body weight, long term therapy for over 5 years, renal disorders, concomitant use with tamoxifen, and macular disease. It can be proposed that the adverse effects of these drugs are dose-dependent. The best way to prevent the incidence of retinopathy was the appropriate dose and duration of treatment of chloroquine and hydroxychloroquine (Marmor et al., 2016).

Some studies have shown that the combination of chloroquine and azithromycin was effective in treating COVID-19 infection. The study that evaluates the QT prolongation as a serious adverse effect and life-threatening from the use of chloroquine and hydroxychloroquine with or without azithromycin has been observed. A recent study by Mercuro et al., infected COVID-19 patients who received combination hydroxychloroquine and azithromycin had a significantly greater $\Delta \mathrm{QT}$ interval than those who received hydroxychloroquine alone. Besides that, the use of loop diuretic as a significant risk factor QTc interval prolongation with adjusted odds ratio was 3.38. Because of QT prolongation, 11\% of patients stopped taking hydroxychloroquine before day 5 . One patient who received combination hydroxychloroquine and azithromycin discontinued due to QT prolongation and developed into ventricular arrhythmia after 3 days of administration of these drugs. The limitation of a study by Mercuro et al., that the patients were in critically ill conditions, received several medications that affect the QT prolongation and increase the cardiotoxic effect, and also this study without a control group (Mercuro et al., 2020). In COVID-19 patients with lung edema, the use of loop diuretic is recommended to treat the edema. The most prevalent side effects of furosemide are hypokalemia. The lower level of potassium in extracellular reduces IKr by enhanced inactivation or exaggerated competitive block by sodium (Numaguchi et al., 2000). Hence, hypokalemia induces the QT interval prolongation. However, the fact that hypokalemia $(<3.0$ $\mathrm{mmol} / \mathrm{L}$ ) increases the blockade of $\mathrm{IKr}$ by the drug is the most important in clinical practice. Correction of hypokalemia properly to the normal or even higher level can shorten the QT interval (Etheridge et al., 2003).

An observational study by van de Broek et al., reported that the QT prolongation was observed in COVID-19 patients with mean QT prolongation of $35 \mathrm{~ms}$ and $34 \mathrm{~ms}$ by computerized and manual interpretation, respectively, after the administration of chloroquine. The dose of chloroquine was $600 \mathrm{mg}$ as a loading dose and followed by $300 \mathrm{mg}$ twice daily, given twelve hours after the administration of loading dose. The total treatment of chloroquine was 5 days. Twentytwo patients have a QT interval of more than $500 \mathrm{~ms}$ during therapy of chloroquine and lead to a higher risk of ventricular arrhythmia. Forty-two of 95 patients had a history of cardiovascular disease (atrial fibrillation, $\mathrm{CAD}, \mathrm{CHF}$ ), and 4 patients used antiarrhythmic drugs (Broek et al., 2020). Several studies stated that the history of cardiovascular disease and concomitant use of an antiarrhythmic drug can worsen the QT prolongation, especially when used with antimalarial drugs, chloroquine or hydroxychloroquine. The finding of QT prolongation by van de Broek et al was much higher than the findings by Mzayek et al. It is because the dose of chloroquine by van de Broek et al was a multiple-dose, meanwhile in Mzayek et al as a single dose of $600 \mathrm{mg}$ chloroquine in healthy subjects (Mzayek et al., 2007). The study by Saleh et al., showed that the maximum QTc during treatment was significantly longer in the combination hydroxychloroquine and azithromycin vs. the monotherapy group (chloroquine) $(470.4 \pm 45.0 \mathrm{~ms}$ 
vs. $453.3 \pm 37.0 \mathrm{~ms}, \mathrm{p}=0.004)$, and also there were no arrhythmogenic deaths during this study (Saleh et al., 2020)

A study by Hancox et al, reported the use of azithromycin dose from twelve case reports and showed that there was no statistically significant relationship between the dose of azithromycin and the duration of QT interval. Risk factors that contribute to the QT prolongation after the administration of azithromycin were female, underlying diseases such as heart disease, older age, coadministration with drugs that induce QT prolongation, bradycardia, and hypokalemia (Hancox et al., 2013). In malaria infection, the use of a combination between chloroquine and azithromycin is to protect against malaria and prevent sexually transmitted infections in pregnancy (Chico et al., 2011). A study by Furst et al., reported that the administration of a loading dose of hydroxychloroquine $400 \mathrm{mg}, 80 \mathrm{mg}$, and $1200 \mathrm{mg} /$ day in patients with rheumatoid arthritis (RA), 8\% of 212 patients had ocular abnormalities, and the adverse ocular events did not occur at six weeks after the administration of this drugs (Furst et al., 1999). A case report by Stas et al., has reported that the conduction disorders and QT prolongation were observed in a patient who taken chloroquine $300 \mathrm{mg}$ /day for 17 years for her SLE. This report indicates that the occurrence of QT prolongation is associated with chloroquine consumption over a long period of time (Stas et al., 2008). Fortunately, therapy of chloroquine or hydroxychloroquine is about five until ten days for COVID-19 infection; therefore, the long treatment is not recommended for COVID-19 infected patients. Numerous studies have been observed, whether the synergism effect of pharmacodynamics between chloroquine and azithromycin was influenced by the pharmacokinetic profile. A study by Cook et al., reported that the combination between azithromycin with a dose $1000 \mathrm{mg}$ and chloroquine, the dose ranging from 300 $\mathrm{mg}$ to $600 \mathrm{mg}$ as chloroquine base in healthy subjects, all parameter pharmacokinetics including $\mathrm{t} \max , \mathrm{C} \max$, AUC, and half-life elimination ( $1 / 2)$ were in the range $80 \%$ until $125 \%$. It can be indicated that the combination of these drugs, there was no clinically pharmacokinetic interaction. It demonstrated that the synergistic effects of the combination between azithromycin and chloroquine are not due to systemic drug-drug interaction (Cook et al., 2006). Because chloroquine and hydroxychloroquine are extensively metabolized in the liver by the cytochrome P450 CYP2D6 enzyme, concomitant administration with other drugs that inhibit the cytochrome P450 CYP2PD6 enzyme, such as antifungal triazole, terbinafine, macrolide, and protease inhibitors cause an increase of chloroquine plasma level and increase the risk of harmful side effects, QT prolongation (Tisdale et al., 2016).
In May 2020, the World Health Organization asked Indonesia to stop the use of chloroquine and hydroxychloroquine in the solidarity trial for the treatment of COVID-19 patients. It is based on the findings of the Solidarity Trial study published on May 22 ${ }^{\text {nd }}, 2020$, in the Lancet Journal. In a study entitled "Hydroxychloroquine or chloroquine with or without a macrolide for treatment of COVID-19: a multinational registry analysis," it was stated that there was an increase in morbidity in COVID-19 patients who received hydroxychloroquine treatment. However, this article has been retracted by the Lancet itself due to conflicting data and poor methodology. Chloroquine and hydroxychloroquine are medical prescriptions only under the supervision of a doctor. In accordance with the approval of its use (emergency use authorization), these drugs are used limited in pandemic conditions for the treatment of adult COVID-19 patients and adolescents with more than $50 \mathrm{~kg}$ of body weight or who are hospitalized. At this time, chloroquine and hydroxychloroquine can still be used in Indonesia in COVID-19 therapy. These drugs are still within safe limits because the dosage is lower, and the duration of use is shorter than the dose in ongoing clinical trials in several countries (BPOM, 2020). The Indonesian Society of Respirology has also stated that COVID-19 patients can still use chloroquine or hydroxychloroquine according to the protocol of management as agreed with the existing dosage with its restrictive rule, as long as outside the research.

\section{CONCLUSION}

Even though the benefits of chloroquine and hydroxychloroquine for COVID-19 therapy are still limited to small clinical trials with poor methodology, unmeasurable outcomes, non-randomized and openlabel, their use can still be considered by assessing the benefits and risks to patients. Clinically, adverse effects of these drugs that induce QT prolongation can be prevented by using usual or recommended dose. From several studies that have been reported the efficacy and safety of chloroquine and hydroxychloroquine, although the data are still very limited, a recommendation is needed to consider using these drugs to treat COVID-19 infections. It is very important to know whether the patient has previously used chloroquine for malaria therapy, or for autoimmune diseases (RA, SLE). Specifically, for patients with autoimmune disease who have been using chloroquine for a long time, consider examining the early signs of cardiac arrhythmia before giving the therapy to patients with COVID-19 infection. The other things to consider are the presence of risk factors that can be precipitated the heart problems related to QT prolongation. These risk factors include concomitant use with azithromycin, patients with a history of cardiac 
arrhythmia, old age, electrolyte disturbances, and concomitant use with loop diuretics.

\section{REFERENCES}

Adelusi, S.A., \& Salako, L.A. (1982). Kinetics of the distribution and elimination of chloroquine in the rat. General Pharmacology, 13, 433-437.

Van den Broek, M.P.H., Möhlmann, J.E., Abeln, B.G.S., Liebregts, M. van Dijk, V.F., \& van de Garde, E. M. W. (2020). Chloroquine-induced QTc prolongation in COVID-19 patients. The Netherlands Heart Journal, 28, 406-409.

Badan Pengawas Obat dan Makanan. (2020). Retrieved from https://www.pom.go.id/new/view/more/ klarifikasi/115/penjelasan Badan POM RI tentang informasi keamanan penggunaan klorokuin dan hidroksiklorokuin pada penyakit new corona virus 2019 . html. access on June 10, 2020.

Borba, M.G.S., Val, F.F.A., Sampaio, V.S. (2020). Effect of high vs low dose of chloroquine diphosphate as adjunctive therapy for patients hospitatalized with severe acute respiratory syndrome coronavirus 2 () SARS-CoV-2) infection: A randomized clinical trial. Jama Network Open. 3(4):e208857. doi:10.1001/ jamanetworkopen.2020.8857.

Chico, R., \& Chandramohan, D. (2011). Azithromycin plus chloroquine: combination therapy for protection against malaria and sexually transmitted infections in pregnancy. Drug Metabolism and Toxicology, 7, 11531167.

Cook, J. A., Randinitis, E. J., Bramson, C. R., \& Wesche, D. L. (2006). Lack of a pharmacokinetic interaction between azithromycin and chloroquine. The American journal of tropical medicine and hygiene, 74(3), 407412.

Cortegiani, A., Ingoglia, G., Ippolito, M., Giarratano, A., \& Einav, S. (2020). A systematic review on the efficacy and safety of chloroquine for the treatment of COVID-19. Journal of Critical Care, 57, 279-283.

Etheridge, S.P., Compton, S.J., Tristani-Firouzi, M., \& Mason, J.W. (2003). A new oral therapy for long QT syndrome: long-term oral potassium improves repolarization in patients with HERG mutations. Journal of the American College of Cardiology, 42(10), 17771782 .
Fang, L., Karakiulakis, G., \& Roth, M. (2020). Are patients with hypertension and diabetes mellitus at increased risk for COVID-19 infection?. The Lancet. Respiratory medicine, 8(4), e21.

Furst, D.E, Lindsley, H., \& Baethge, B. (1999). Doseloading with hydroxychloroquine improves the rate of response in early, active rheumatoid arthritis: a randomized, double-blind six-week trial with eighteenweek extension. Arthritis \& Rheumatology, 42 (2), 357365 .

Jorge, A., Ung, C., Young, L.H., Melles, R.B., \& Choi, H.K. (2018). Hydroxychloroquine retinopathyimplications of research advances for rheumatology care. Nature Reviews Rheumatology, 14(12), 693-703.

Gao, J., Tian, Z., \& Yang, X. (2020). Breakthrough: Chloroquine phosphate has shown apparent efficacy in treatment of COVID-19 associated pneumonia in clinical studies. BioScience Trends, 14(1):72-73.

Gautret, P., Lagier, J. C., Parola, P., Hoang, V. T., Meddeb, L., Mailhe, M., Doudier, B., Courjon, J., Giordanengo, V., Vieira, V. E., Tissot Dupont, H., Honoré, S., Colson, P., Chabrière, E., La Scola, B., Rolain, J. M., Brouqui, P., \& Raoult, D. (2020). Hydroxychloroquine and azithromycin as a treatment of COVID-19: results of an open-label non-randomized clinical trial. International Journal of Antimicrobial Agents, 56(1), 105949.

Gruppo collaborativo COVID-19 Lombardia. (2020). Vademecum per la cura delle persone con malattia da COVID-19 Edizione 2.0, 13 marzo 2020. Retrieved from http://www.simit.org/m

Guo, Y.R., Cao, Q.D., \& Hong, Z.S. (2020). The origin, transmission and clinical therapies on coronavirus disease 2019 (COVID-19) outbreak - an update on the status. Military Medical Research, 7(1), 11.

Han, Y., \& Yang, H. (2020). The transmission and diagnosis of 2019 novel coronavirus infection disease (COVID-19): A Chinese perspective. Journal of Medical Virology, 92(6), 639-644.

Hancox, J. C., Hasnain, M., Vieweg, W. V., Crouse, E. L., \& Baranchuk, A. (2013). Azithromycin, cardiovascular risks, QTc interval prolongation, torsade de pointes, and regulatory issues: A narrative review based on the study of case reports. Therapeutic Advances in Infectious Disease, 1(5), 155-165. 
Indonesian Society of Respirology. (2020). Tatalaksanana pasien COVID-19. Jakarta: PDPI.

Kalia,S., \& Dutz, J.P.(2007). New concepts in antimalarial use and mode of action in dermatology. Dermatologic Therapy, 20(4), 160-174.

Kementerian Kesehatan Republik Indonesia. (2020). Info Infeksi Emerging Kementerian Kesehatan RI. [updated 2020 May 21]. Retrieved from: https:// infeksiemerging. kemkes.go.id/

Lee, S.J., Silverman, E., \& Bargman, J.M. (2011). The role of antimalarial agents in the treatment of SLE and lupus nephritis. Nature Reviews Nephrology, 7(12), 718729.

Li, X., Geng, M., Peng, Y., Meng, L., \& Lu, S. (2020). Molecular immune pathogenesis and diagnosis of COVID-19. Journal of Pharmaceutical Analysis, 10(2), 102-108.

Liu, J., Cao, R., Xu, M., Wang, X., Zhang, H., Hu, H., Li, Y., Hu, Z., Zhong, W., \& Wang, M. (2020). Hydroxychloroquine, a less toxic derivative of chloroquine, is effective in inhibiting SARS-CoV-2 infection in vitro. Cell Discovery, 6, 16.

Lu, R., Zhao, X., Li, J., Niu, P., Yang, B., Wu, H., Wang, W., Song, H., Huang, B., Zhu, N., Bi, Y., Ma, X., Zhan, F., Wang, L., Hu, T., Zhou, H., Hu, Z., Zhou, W., Zhao, L., Chen, J., ... \& Tan, W. (2020). Genomic characterisation and epidemiology of 2019 novel coronavirus: implications for virus origins and receptor binding. Lancet, 395(10224), 565-574.

Marmor, M.F., Kellner, U., Lai, T.Y., Melles, R.B., \& Mieler, W.F. (2016). Recommendations on screening for chloroquine and hydroxychloroquine retinopathy (2016 Revision). Ophthalmology, 123, 1386-1394.

Mercuro, N. J., Yen, C. F., Shim, D. J., Maher, T. R., McCoy, C. M., Zimetbaum, P. J., \& Gold, H. S. (2020). Risk of QT Interval Prolongation Associated With Use of Hydroxychloroquine With or Without Concomitant Azithromycin Among Hospitalized Patients Testing Positive for Coronavirus Disease 2019 (COVID-19). JAMA Cardiology, e201834.

Mzayek, F., Deng, H., \& Mather, F.J. (2007). Randomized dose-ranging controlled trial of AQ-13, a candidate antimalarial,and chloroquine in healthy volunteers. PLoS Clinical Trials, 2, 1-15.
National Health Commission \& State Administration of Traditional Chinese Medicine. (2020). Diagnosis and Treatment Protocol for Novel Coronavirus Pneumonia (Trial Version 7) (Released by on March $3^{\text {rd }}, 2020$ ). Retrieved from https://www. chinalawtranslate.com/wpcontent/uploads/2020/03/Who-translation.pdf

Numaguchi, H., JohnsonJr, J.P., Petersen, C.I., \& Balser, J.R. (2000). A sensitive mechanism for cation modulation of potassium current. Nature Neuroscience, 3(5), 429-430.

Pae, C.U. (2015). Why systematic review rather than narrative review. Psychiatry Investigation, 12(3), 417419.

Saleh, M., Gabriels, J., Chang, D., Soo Kim, B., Mansoor, A., Mahmood, E., Makker, P., Ismail, H., Goldner, B., Willner, J., Beldner, S., Mitra, R., John, R., Chinitz, J., Skipitaris, N., Mountantonakis, S., \& Epstein, L. M. (2020). Effect of Chloroquine, Hydroxychloroquine, and Azithromycin on the Corrected QT Interval in Patients With SARS-CoV-2 Infection. Circulation: Arrhythmia and Electrophysiology, 13(6), e008662.

Savarino, A., Boelaert, J.R,. Cassone, A., Majori, G., \& Cauda, R. (2003). Effects of chloroquine on viral infections: an old drug against today's diseases?. The Lancet Infectious Diseases, 3, 722-727.

Srinivasa, A., Tosounidou, S., \& Gordon, C. (2017). Increased incidence of gastrointestinal side effects in patients taking hydroxychloroquine: a brand-related issue?. The Journal of Rheumatology, 44, 398.

Stas, P., Faes, D., \& Noyens, P. (2008). Conduction disorder and QT prolongation secondary to long-term treatment with chloroquine. International Journal of Cardiology, 127(2), e80-e82.

Tisdale, J.E. (2016). Drug-induced QT interval prolongation and torsades de pointes: Role of the pharmacist in risk assessment, prevention, and management. Canadian Pharmacists Journal (Ott), 149(3), 139-152.

Touret, F., \& de Lamballerie, X. (2020). Of chloroquine and COVID-19. Antiviral Research, 177, 104762.

Wang, M., Cao. R., Zhang, L., Yang, X., Liu, J., \& Xu, M. (2020). Remdesivir and chloroquine effectively inhibit the recently emerged novel coronavirus (2019$\mathrm{nCoV}$ ) in vitro. Cell Research, 30, 269-271. 
World Health Organization. (2020). Coronavirus disease 2019 (COVID-19) Situation Report 74. Geneva: WHO

Yao, X., Ye, F., Zhang, M., Cui, C., Huang, B., Niu, P., Liu, X., Zhao, L., Dong, E., Song, C., Zhan, S., Lu, R., Li, H., Tan, W., \& Liu, D. (2020). In Vitro Antiviral Activity and Projection of Optimized Dosing Design of Hydroxychloroquine for the Treatment of Severe Acute Respiratory Syndrome Coronavirus 2 (SARS-CoV-2). Clinical infectious diseases : an official publication of the Infectious Diseases Society of America, ciaa237

Zumla, A., Hui, D.S., Azhar, E.I., Memish, Z.A., \& Maeurer, M. (2020). Reducing mortality from 2019nCoV: host-directed therapies should be an option. Lancet, 395(10224), 35-36. 